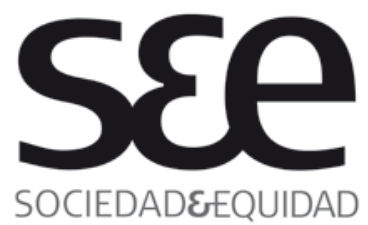

\section{Migración de retorno, familias transnacionales y demandas educativas}

\author{
Return migration, transnational families and \\ educational demands
}

\author{
Nombre: \\ Filiación: \\ País: \\ Correo:
}

\author{
Luis Fernando Ocampo Marín (1) \\ Universidad Autónoma de Guerrero \\ México \\ fer7117@gmail.com
}

\title{
Resumen
}

La migración de México hacia Estados Unidos es crecientemente familiar. Esto implica que en los últimos años menores y adolescentes nacidos en México y en Estados Unidos se mueven entre los dos países con demandas educativas. La migración de retorno causada por la crisis del mercado laboral en Estados Unidos, o por la deportación debido a su condición de ilegalidad, crean nuevos escenarios y actores que complejizan la sociedad mexicana. La presencia creciente de estudiantes con antecedentes y experiencia educativa, social, cultural y familiar diferenciada, trae consigo identidades cambiantes y visiones comparativas que dificultan su integración social y rendimiento escolar, y les sumergen en estados de disonancia cognitiva. El desafío para una escuela ajena a sus necesidades es grande y trasciende fronteras. El artículo destaca la ineludible vinculación de estos/as estudiantes con la de su contexto familiar transnacional. El trabajo de campo se centró en la experiencia de la escuela en donde confluyen los/as estudiantes binacionales, personal administrativo, profesores/as, padres de familia y responsables del Programa Binacional de Educación Migrante México - Estados Unidos. La investigación es exploratoria con una metodología inductiva. Los hallazgos son analíticamente observados bajo el enfoque transnacional y proveen de referentes generales a la problemática estudiada.

\section{Palabras Claves}

Migración de retorno, Familias transnacionales, Estudiantes transnacionales, Demandas educativas, Identidades cambiantes, Disonancia cognitiva.

\footnotetext{
${ }^{1}$ El autor es Doctor en Economía Política. Sus líneas de investigación son: migración y estudios del territorio.
} 


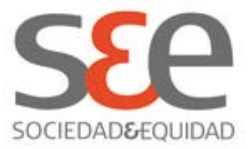

\begin{abstract}
Migration from Mexico to the United States is increasingly familiar. This indicates that in recent years children and adolescents born in Mexico and the United States move between the two countries to satisfy their educational demands. Return migration, caused by the crisis in the labor market in the United States, or by deportation due to their illegal status, creates new scenarios and 'actors' that causes more complex problems in Mexican society. The increasing presence of students with educational experience, but with a differentiated social, cultural and familial background, brings changing identities and comparative visions that impede their social integration and school performance, and merge them in a cognitive dissonance state. The challenge for the school to meet is considerable and transcends national boundaries. This paper highlights the inescapable link between these students with their transnational family context. The field work was based on the school experience, in which students, school administration, teachers, parents, and representantes of the "Programa Binacional de Educación Migrante México Estados Unidos", came together. This exploratory research uses an inductive methodology. The findings are analyzed under a transnational focus and provide us with general references regarding the studied problems.
\end{abstract}

\title{
Keywords
}

Return migration, Transnational families, Transnational students, Educational demands, Changing identities, Cognitive dissonance.

\section{Introducción}

La Secretaría de Educación Pública SEP - Guerrero a través del Programa Binacional de Educación Migrante México - Estados Unidos de América (PROBEM) emprendió en el año 2010 una investigación sobre estudiantes binacionales en el estado de Guerrero. El estudio se enfoca en la experiencia de la escuela en donde confluyen el Programa (PROBEM) y los alumnos y alumnas binacionales. Aunque el trabajo de campo se centra en un estado de México, el marco analítico, la metodología, los resultados, las conclusiones y propuestas sirven de referentes generales a la problemática estudiada. Lo que se expone son algunas consideraciones conceptuales y hallazgos parciales de puntos que merecen mayor estudio y consideración y que solo pretende ser de carácter exploratorio de una realidad creciente que no se puede ignorar. 


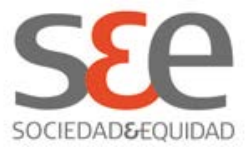

\section{El Problema}

La decisión de emigrar a Estados Unidos como estrategia de superar la precariedad económica familiar, induce a que los hijos e hijas migren con sus padres. Salen de México por decisión de los padres y regresan a México por la misma razón, ellos/as no tienen otra alternativa que acompañarlos en estas dinámicas de trabajo. Estos niños y niñas siguen a sus padres en su movilidad que en muchos casos es repetitiva y bidireccional.

La experiencia migratoria de estos niños, niñas y adolescentes se da en un contexto de dispersión afectiva familiar y de extrema fragilidad legal y riesgo fronterizo. Su representación de esta movilidad tiene un carácter transitorio determinado por su condición jurídica y amenaza de deportación.

El retorno a México de familias migrantes provenientes de Estados Unidos de América ha generado una particular población en las escuelas mexicanas de estudiantes con experiencia educativa en el sistema escolar norteamericano. Su perfil es amplio, unos salen de México con algunos años de escuela, otros muy pequeños cursan sus primeros estudios en Estados Unidos; unos nacen y estudian varios años en la Unión Americana, otros cruzan la frontera varias veces y asisten a la escuela intercaladamente entre México y diferentes estados de Norteamérica.

A estos niños/as y jóvenes migrantes de retorno inscritos en primaria y secundaria en el sistema escolar mexicano con experiencia educativa en Estados Unidos de América, son los que, en este estudio, llamamos estudiantes transnacionales o alumnos/ as binacionales.

\section{Metodología}

Dado que los actores principales son los y las jóvenes estudiantes migrantes de retorno en situación familiar transnacional, este estudio toma en cuenta una referencia espacial múltiple, que incluya tanto el espacio de partida como el espacio de llegada, centrando la atención en los procesos migratorios en sí mismos, sobre el funcionamiento social de un sistema de movilidad establecido y creado por los y las migrantes en su conjunto.

La metodología de la investigación es la combinación de componentes cuantitativos y cualitativos para la obtención de nueva información. En el referente cuantitativo, la investigación se llevó en función de las unidades escolares con incidencia de alumnos binacionales ubicadas en los municipios de mayor concentración escolar. La selección se realizó en base a un diseño semialeatorio simple, en donde se relacionaron el número de estudiantes escuela con el municipio y se determinó las escuelas en donde se aplicarían los instrumentos de evaluación necesarios. 


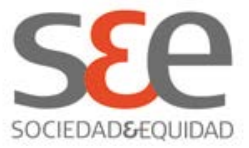

Para la ubicación escalonada y rápida de los individuos a estudiar, se usó la metodología llamada bola de nieve (SNOWBALL), que permitió después de la entrevista y con la ayuda de el/la estudiante ubicar a un/a profesor/a relacionado/a con la problemática. A su vez, el/la profesor/a nos remitía a otros colegas o al personal administrativo de la escuela, que a su vez nos relacionaba con padres y madres de familia, que también nos remitían a otros padres y madres posibles de entrevistar. Igual sucedió en las oficinas regionales del PROBEM con el personal administrativo.

En lo cualitativo, en el trabajo de campo se aplicaron entrevistas a profundidad semi - estructuradas al personal administrativo del Programa PROBEM, a directivos, docentes, estudiantes, padres y madres de familia, que hayan sido objeto del Programa con el fin de detectar los principales problemas que enfrentan, y analizar las necesidades de los niños/ as y jóvenes migrantes, no sólo educativos, sino también de tipo afectivo y social.

El estudio al ser básicamente exploratorio de un fenómeno reciente, se guía por una metodología inductiva. No obstante, la descripción de resultados son analíticamente observados bajo el enfoque transnacional, que en este caso da cuenta de las familias y alumnos/as transnacionales de retorno en el contexto del sistema educativo mexicano.

\section{Resultados}

Los resultados en lo posible, se presentan diferenciados (primaria/secundaria) dado que la problemática presenta cierta especificidad según el nivel de estudios. No es lo mismo nacer o vivir y estudiar desde la tierna infancia en Estados Unidos de América, que emigrar después de haber cursado algunos años en México. Retornar a México y readaptarse al sistema escolar, no siempre es favorable, especialmente para los y las adolescentes que estudian secundaria y poseen actitudes y capacidades diferentes a la niñez.

La presencia creciente de estudiantes en niveles de primaria y secundaria con antecedentes y experiencia educativa, cultural y familiar diferenciada, trae consigo identidades cambiantes y visiones comparativas que sumergen al estudiante en estados de disonancia cognitiva y dificultan su integración social y rendimiento escolar.

\section{Discusión y hallazgos}

Los estudios de la migración internacional se han enfocado básicamente en torno al tema de remesas y desarrollo, descuidando muchos otros tópicos implicados. Recientemente, se ha introducido el enfoque de género pero bajo el mismo referente de remesas y desarrollo. Por otro lado acompañados de la categoría del transnacionalismo, se han vertido análisis de comunidades y 


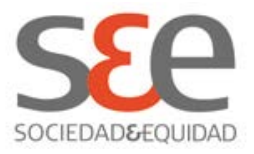

familias transnacionales. Sin embargo hay muy pocos estudios de alumnos y alumnas transnacionales, y menos vinculados a sus particulares contextos y formas familiares. Estos estudios no abordan el caso de la migración de retorno de estudiantes con experiencia educativa en Estados Unidos de América en un marco conceptual propio de sus familias y de la complejidad de una escuela ajena a sus necesidades.

La investigación permitió visualizar en México un nuevo actor social que emerge de la particular migración y relación laboral con un país limítrofe. Su experiencia educativa, su geografía emocional, su paisaje familiar y su enfoque interpretativo, surgen con fuerza diferenciada a su retorno obligado, en una escuela ajena a la diversidad y reproductora de identidad nacional. Ellos/ as son la primera generación de la diversidad en el seno de un sistema escolar que los/as invisibiliza y obliga a integrarse a cambio de autonegación, riesgo de exclusión y fracaso escolar.

El desafío para la escuela es grande y trasciende fronteras. Obliga a producir insumos e información básica con una visión transnacional y basada en las prácticas de los propios actores y actoras sociales, que oriente la toma de decisiones en la planeación de estrategias de atención educativa.

En este artículo destacamos la ineludible vinculación entre las problemáticas educativas de estos alumnos y alumnas con la de su contexto familiar transnacional.

El artículo se organiza en tres partes:

- El enfoque transnacional.

- Estudiantes binacionales.

- El retorno escolar.

\section{EL ENFOQUE TRANSNACIONAL}

El enfoque transnacional en el estudio de los procesos migratorios internacionales nace a finales de los años 80 y principios de los 90 , referido al conjunto de procesos por los cuales los inmigrantes crean y mantienen relaciones sociales multitrenzadas que vinculan las sociedades de origen y de destino (Basch et al., 1994; Guarnizo, 2006; Schiller et al., 1992; Faist, 2000; Bryceson y Vuorela, 2002).

Este nuevo paradigma desde el que se teoriza la movilidad humana en sociedades contemporáneas, concibe la migración no como un proceso 


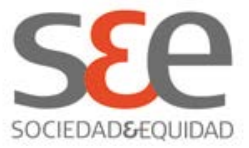

dicotómico (unidireccional o bidireccional), sino dinámico de construcción y reconstrucción de redes sociales que estructuran la movilidad espacial y la vida laboral, social, cultural y política, tanto de la población migrante como de familiares, amigos y comunidades en los países de origen y de destino o destinos (Guarnizo, 2006).

La visión transnacional da un mayor protagonismo a las articulaciones y conexiones que se dan en las migraciones contemporáneas, como son las redes migratorias transnacionales, y las familias transnacionales.

Desde la epistemología, permite superar el "nacionalismo metodológico", como aquella tendencia a aceptar el Estado-nación y sus fronteras como un elemento dado y limitador en el análisis social (Wimmer y Glick Schiller, 2003). Como consecuencia se opta por un enfoque en donde la familia transnacional es en sí misma el lugar de pertenencia e identidad. El contenedor en donde acontece la familia transnacional ya no es el mismo al del Estado Nación. La familia ya no es un anclaje ni eslabón en la justificación, recreación, y construcción del Estado Nación. La frontera se desvanece, y con ella el control ¿patriótico?.

La identidad familiar adquiere preponderancia sobre lo nacional que tiende a desvanecerse (Giménez, 2010). La representación simbólica de su identidad colectiva o mexicanidad se debilita para reforzar su núcleo familiar de pertenencia y membrecía básica. El gesto patriótico se horada, tal y como lo refieren los padres, madres y estudiantes en situación de familia transnacional entrevistados. Los primeros al desear que sus hijos/hijas puedan regresar y continuar o retomar sus estudios, y los segundos al manifestar una identidad compartida entre Estados Unidos de América y México, o sentirse más americano/a que mexicano/a.

\section{La Familia transnacional}

Sin excepción los/as estudiantes, padres y madres de familia entrevistados se refieren a su familia en situación de movilidad entre México y Estados Unidos de América, y enfatizan la dispersión o dislocación de sus miembros centrales distribuidos entre estos dos Estados. La ocupación simultánea o pendular de dos Estados nación es una característica básica del fenómeno de las familias transnacionales. Esto implica que los procesos y las relaciones entre las personas definidas como parientes, adquieran diferentes modalidades de reagrupación vinculadas a múltiples formas de integrarse en la sociedad receptora y de mantener la relación entre su parentela y el país de origen (Hondagneu - Sotelo \& Ávila, 1997; Pribilsky, 2004). 


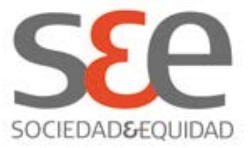

Aunque el hecho migratorio en sí mismo fragmenta a la familia en el tiempo y el espacio, ésta mantiene y recrea los vínculos a través de las fronteras, evitando la dispersión, desintegración o ruptura (Pedone, 2007).

Las relaciones que se construyen entre los miembros de estas familias generan nuevas formas de cuidado y de entender la maternidad y la paternidad, que cuestionan las concepciones de familia asociada a la coresidencia y la presencialidad, y contrastan con el esquema de familia tradicional, normal, regulada y aceptada.

La nueva configuración familiar implica reformulación de las dinámicas de poder y nuevas asimetrías en su ejercicio. Un rol más activo de la presencia de las mujeres (madres, hijas, tías, abuelas, comadres) que actúa como magnetismo vinculante y entre las partes, tanto al interior del núcleo familiar como en los espacios sociales y económicos.

El papel vinculante se construye a través de redes familiares y sociales que contribuyen a la disminución simbólica de la distancia espacial y cultural que los separa. A la par de las remesas, las estrategias vinculantes permiten que sus miembros se sientan parte de una unidad y perciban su bienestar desde una dimensión colectiva, a pesar de la distancia física. Estas llamadas "remesas sociales" son múltiples: redes sociales, fotografías, cartas, llamadas telefónicas, regalos, tarjetas recordatorias, rituales como bodas, compromisos, fiestas, bautizos, santos patronos, la organización de las reuniones por vacaciones e incluso visitas esporádicas del migrante (Di Leonardo, 1992; Bryceson \& Vuroela, 2002).

De acuerdo con Levitt (1998 y 2001), las "remesas sociales", definidas como el conjunto de valores sociales y culturales, estilos de vida, pautas de comportamiento y capital social que se da entre las comunidades de origen y de destino, constituyen beneficios intangibles a largo plazo, al transferirse conocimientos $y$ habilidades mediante actividades comunicativas 0 interacciones cara a cara entre personas que sostienen algún tipo de vínculo.

Parella y Cavalcanti (2006: 251), afirman que "sin lugar a dudas, uno de los tipos de práctica transnacional más relevante, con mayores repercusiones en las vidas de los migrantes y sus familias, es la materialización de las conexiones transnacionales a través de las remesas monetarias y sociales". Si las remesas monetarias afectan las actividades económicas y de trabajo de los que la reciben, las remesas sociales influyen en la construcción identitaria de las personas involucradas, y pueden generar cambios de orden político, social y cultural. 


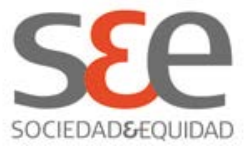

En el caso de las familias transnacionales que nos ocupa, se caracterizan por la precariedad legal que las obliga a retornar. Esto refuerza su situación de movilidad y transitoriedad y crea una auto - narrativa familiar que asume el hecho de la migración y separatividad como temporales ya sea por su reunificación en la sociedad receptora o por su definitivo regreso.

Esta auto - narrativa, reproduce una tensión - negociación entre la diferenciación y asimilación con la sociedad receptora. La ambivalencia se presenta, como lo confirma este estudio, de manera desigual al interior de sus miembros, y puede llegar a ser fuente de conflicto entre unos hijos más permeables a la mutación cultural y social y unos padres blindados a ellas.

Para actualizar y reforzar su identidad colectiva, las familias transnacionales recrean en la sociedad receptora, rituales de su entorno cultural y religioso de procedencia: fiestas tradicionales, música y comidas autóctonas, historias fundacionales de su comunidad, uso reiterado en su lenguaje de modismos o frases propias, realización de tareas hogareñas, gustos y reproducción estética en su forma de vestir y decorar a la usanza de su tierra natal, continuidad en sus sistemas de creencias religiosas y sobre el proceso de salud enfermedad. Estas dinámicas Ilegan a fundar un entorno urbano de calles y colonias en donde se recrea económica, social y estéticamente el lugar que los vio nacer.

Esta novedosa configuración familiar, resultado del nuevo patrón migratorio México - Estados Unidos de América, formula una nueva unidad de análisis y desafíos teóricos y sociales. El hecho de la incorporación de las mujeres al proceso migratorio y por ende al reagrupamiento familiar que trae consigo, altera no solo lo que culturalmente se entiende por mujer - esposa - madre, sino también otros aspectos de la cultura que individuos y familias llevan con ellos cuando migran. El resultado es una deconstrucción - construcción de lo que tradicionalmente se entiende por familia.

\section{ESTUDIANTES BINACIONALES}

A partir de la década de los noventa, cambió el modelo migratorio tradicional de México a Estados Unidos. La reforma migratoria "Inmigration Reform and Control Act" (IRCA) aprobada en Estados Unidos en noviembre de 1986, legalizó a más de dos millones y medio de indocumentados de los cuales el 43 por ciento fueron mujeres. Con esto se legalizó el orden familiar y permitió su reunificación (Durand y Massey, 2003; Woo, 2001). Ahora, ya no se trata únicamente de una migración de adultos, particularmente de hombres, sino que también una femenina y familiar, y por ende de niños, niñas y jóvenes inscritos en los sistemas educativos de uno o de los dos países en busca de superación académica. 
Como referente contextual y de acuerdo a Estadísticas Generales de Población, de la Oficina de Censo de Los Estados Unidos de América (en inglés: Current Population Survey (CPS), US. Census Bureau), citadas en Fundación BBVA (2012: 35 cuadro 10), para el año 2008 el total de mexicanos en Estados Unidos, ascendían a 30.7 millones, de los cuales 18.9 millones son de segunda y tercera generación, y 11.8 millones son personas nacidas en México, de los cuales 6.9 millones no contaban con documentos.

El incremento de la migración reciente de retorno causada por la crisis 2007 2009 del mercado laboral en Estados Unidos, o por la deportación debido a su condición de ilegalidad, o por contextos propios familiares (cuadro 1), ha generado en México una presencia mayor de estudiantes en niveles de primaria y secundaria con antecedentes educativos diferenciados que exige una atención basada en las experiencias y prácticas de los propios actores y actoras sociales (cuadro 2).

\begin{tabular}{|c|c|c|c|c|}
\hline \multicolumn{5}{|c|}{ Cuadro 1: Migración de retorno, estimaciones de entradas a México } \\
\hline Fuente & período & $\begin{array}{l}\text { Miles en } \\
\text { el } \\
\text { período }\end{array}$ & $\begin{array}{l}\text { Promedio } \\
\text { por año }\end{array}$ & Descripción \\
\hline $\begin{array}{l}\text { Department of } \\
\text { Homeland } \\
\text { Security }\end{array}$ & $2007-2010$ & 340 & 85 & $\begin{array}{l}\text { Reducción de migrantes } \\
\text { mexicanos indocumentados en } \\
\text { EE UU }\end{array}$ \\
\hline $\begin{array}{l}\text { Pew Hispanic } \\
\text { Center }\end{array}$ & $2007-2010$ & 500 & 125 & $\begin{array}{l}\text { Reducción de migrantes } \\
\text { mexicanos indocumentados en } \\
\text { EE UU }\end{array}$ \\
\hline \multirow[t]{2}{*}{ ENOE (INEGI) } & $2006-2008$ & 1220 & 407 & \multirow[t]{2}{*}{$\begin{array}{l}\text { Entradas de mexicanos de } \\
\text { diferentes países }\end{array}$} \\
\hline & $2009-2011$ & 780 & 260 & \\
\hline \multirow{3}{*}{$\begin{array}{l}\text { EMIF Norte } \\
\text { (INEGI) }\end{array}$} & $2002-2004$ & 1209 & 403 & \multirow[t]{3}{*}{ Entradas de EE UU } \\
\hline & $2005-2007$ & 1023 & 341 & \\
\hline & $2008-2010$ & 1087 & 362 & \\
\hline \multirow[t]{2}{*}{ Censo (INEGI) } & $1995-2000$ & 528 & 106 & \multirow[t]{2}{*}{ Entradas de diferentes países } \\
\hline & $2005-2010$ & 1293 & 259 & \\
\hline \multicolumn{5}{|c|}{$\begin{array}{l}\text { Fuente: Fundación BBVA, Bancomer y BBVA (2012), research con datos de diferentes } \\
\text { fuentes. }\end{array}$} \\
\hline
\end{tabular}


De acuerdo con la Fundación BBVA, Bancomer y el Servicio de Estudios Económicos, México de BBVA Research (2012), el Departamento de Seguridad Nacional de los Estados Unidos de América (en inglés: Departmen of Home Land Security) y el Centro de Investigación Pew (en inglés: Pew Spanic Center) especializado en investigación sobre temas de población hispana en Estados Unidos tanto nativa como migrante, incluyendo la mexicana, confirman la reducción de migrantes mexicanos indocumentados en EE UU (cuadro 1).

En México, la Encuesta Nacional de Ocupación y Empleo (ENOE) del Instituto Nacional de estadística y Geografía (INEGI), con datos trimestrales a escala nacional y una muestra de 120 mil hogares y más de 400 mil personas, señala en el períodos 2005 - 2007, una migración de retorno promedio por año de 420 mil migrantes mexicanos (cuadro 2). En el cuadro 1, se observa la diferencia entre los períodos 2006 - 2008 y 2009 - 2011 en donde el número de inmigrantes es casi el doble en el primer caso.

\begin{tabular}{|c|c|c|c|c|c|c|}
\hline \multicolumn{7}{|c|}{ Cuadro 2 } \\
\hline \multirow{2}{*}{ Año } & \multicolumn{5}{|c|}{ Grupos de edad } \\
\cline { 2 - 7 } & $\mathbf{0}$ a 17 a 34 & $\mathbf{3 5}$ a 39 & $\mathbf{5 0}$ a 64 & $\mathbf{6 5}$ a más & $\begin{array}{c}\text { Total } \\
\text { (miles) }\end{array}$ \\
\hline 2005 & 7.9 & 56.3 & 24.5 & 7.4 & 3.9 & 425 \\
\hline 2006 & 6.7 & 57.1 & 26.9 & 7.4 & 1.8 & 437 \\
\hline 2007 & 6.9 & 56.1 & 27.9 & 7.6 & 1.5 & 403 \\
\hline 2008 & 6.9 & 57.7 & 25.8 & 7.7 & 2.0 & 379 \\
\hline 2009 & 8.8 & 52.6 & 28.7 & 7.0 & 2.9 & 329 \\
\hline 2010 & 8.2 & 54.6 & 26.7 & 8.5 & 2.0 & 264 \\
\hline 2011 & 6.2 & 56.5 & 22.3 & 11.3 & 3.7 & 202 \\
\hline $\begin{array}{l}\text { Fuente: Fundación BBVA, Bancomer y BBVA (2012), research, estimaciones a partir de } \\
\text { la construcción de páneles de la ENOE, } 2005-2011, \text { con la metodología de migrantes } \\
\text { internacionales del INEGI. }\end{array}$ & & & & \\
\hline
\end{tabular}




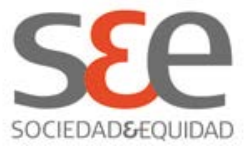

En el cuadro 2 se observa de forma nítida el incremento en el 2009 y 2010 del retorno del grupo de edad de 0 a 17 años, al pasar de representar el $6.9 \%$ en el 2008 , al $8.8 \%$ en el 2009 . Niños y jóvenes cruzan la frontera invisibilizados por una lógica institucional y académica preocupada fundamentalmente por las remesas y sus "beneficios" económicos, ignorando el factor humano y la crisis social que trae consigo esta gran movilidad obligada.

Estos/as nuevos/ as actores/ as producto de la migración de retorno han complejizado la problemática educativa en México y obligan a producir insumos e información básica que orienten a la toma de decisiones en la planeación de estrategias de atención educativa con una visión transnacional. Tanto la Organización de las Naciones Unidas para la Educación la Ciencia y la Tecnología (UNESCO), como la Organización de las Naciones Unidas (ONU) han expresado estas preocupaciones relacionadas con la cultura y la educación en contextos de gran movilidad internacional de la población mundial. La UNESCO en su manifestación mundial sobre educación que presentó en París el 9 de Octubre de 1998, exhortó a cultivar la cooperación interinstitucional y el intercambio académico para brindar experiencias internacionales en la formación de los estudiantes, todo ello, proporcionando un trato con igualdad de los conceptos de calidad en la educación, la equidad, el acceso y cooperación académica internacional. La ONU en su Convención sobre Derechos de la Niñez en 1988 y ratificada por México el 21 de septiembre de 1990, establece el derecho a la educación que tienen todos los niños y niñas sin distinción alguna por motivos de raza, color, sexo, idioma, religión, opinión política 0 de otra índole, origen nacional o social, posición económica, nacimiento o cualquier otra condición.

Los esfuerzos de colaboración entre los Sistemas Escolares Estatales entre México y Estados Unidos de América dieron origen al Programa Binacional de Educación Migrante México - Estados Unidos de América (PROBEM) en el año de 1982. El estado de Guerrero a partir de 1996 participa dentro de esta estrategia. De acuerdo a le Secretaría de Educación del estado de Guerrero (2010), en el período 2005 - 2006, en el estado, se atendió una población de 510 estudiantes centrados mayoritariamente en el nivel primaria (83.7\%), puesto que únicamente el $16.3 \%$ se hallaban en nivel secundaria. Para el período 2008 - 2009, la matrícula se incrementó un $27.66 \%$ al pasar de 510 a 651 estudiantes, con un peso representativo en ascenso respecto al período anterior del nivel secundario del $27.65 \%$ y un menor peso del nivel de primaria (72.35\%) (Cuadro 3). 


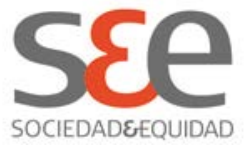

\begin{tabular}{|c|c|c|c|c|c|c|c|}
\hline \multicolumn{8}{|c|}{$\begin{array}{l}\text { Estudiantes de primaria y secundaria atendidos en el estado de Guerrero, México } \\
\text { por el PROBEM. Ciclo escolar } 2005 \text { - } 2006 \text { y } 2008 \text { - } 2009\end{array}$} \\
\hline \multicolumn{4}{|c|}{ Estudiantes atendidos } & \multicolumn{4}{|c|}{ Porcentaje: variación } \\
\hline \multicolumn{2}{|c|}{ Primaria } & \multicolumn{2}{|c|}{ Secundaria } & \multicolumn{2}{|c|}{ Primaria } & \multicolumn{2}{|c|}{ secundaria } \\
\hline $\begin{array}{l}2005- \\
2006\end{array}$ & $\begin{array}{c}2008- \\
2009\end{array}$ & $\begin{array}{l}2005- \\
2006\end{array}$ & $\begin{array}{c}2008- \\
2009\end{array}$ & $\begin{array}{l}2005- \\
2006\end{array}$ & $\begin{array}{l}2008- \\
2009\end{array}$ & $\begin{array}{c}2005- \\
2006\end{array}$ & $\begin{array}{l}2008- \\
2009\end{array}$ \\
\hline $\begin{array}{c}427 \\
(83.7 \%)\end{array}$ & $\begin{array}{c}471 \\
(72.4 \%)\end{array}$ & $\begin{array}{c}83 \\
(16.3 \%)\end{array}$ & $\begin{array}{c}180 \\
(27.6 \%)\end{array}$ & 100 & $10.3 \%$ & 100 & $116.9 \%$ \\
\hline Fuente: $\mathrm{e}$ & oración p & COn & ión & 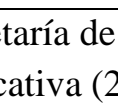 & $\begin{array}{l}\text { ucació } \\
\text { )). }\end{array}$ & 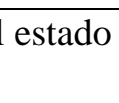 & \\
\hline
\end{tabular}

La mayor variación porcentual entre los dos ciclos se observó en el nivel de secundaria que tuvo un incremento de $116.9 \%$ frente a un $10.3 \%$ del nivel de primaria. Estas cifras son significativas ya que corroboran el incremento de estudiantes y familias de retorno coincidentemente con el comienzo de la crisis del mercado laboral 2007 - 2009 en Estados Unidos de América (Cuadro 3).

En relación al destino en los Estados Unidos de América de donde provienen los estudiantes migrantes al estado de Guerrero en el ciclo escolar 2008 - 2009, el peso recae en el estado de California con $30.63 \%$ le sigue muy distante el estado de Texas con el $9.11 \%$ Georgia con el $8.91 \%$ Carolina del Norte con el 7.33\% Illinois con el $6.24 \%$ Arizona con el $5.64 \%$ Florida con el $4.65 \%$ y en muy menor proporción los estados de Colorado 3.37\% y Washington $2.77 \%$ para dejar el resto de los estados con muy poca presencia (SEG 2010).

\section{EL RETORNO ESCOLAR}

El retorno de estudiantes tras una estancia educativa y una situación ilegal en Estados Unidos, empieza con las dificultades de los trámites escolares de revalidación ante la Secretaría de Educación. La impronta que el estudiante tiene de la escuela en México la vive desde una visión comparativa con el referente escolar norteamericano. Su educación y cultura diferenciada le crean problemas de inserción y conflictos identitarios frente a un sistema impedido para la diferencia. Esta situación aunada a su vivencia íntima de ser parte de 


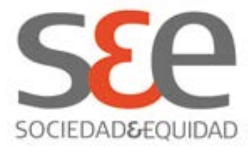

una familia transnacional, lo sumerge en estados de disonancia cognitiva que fragilizan su personalidad y propician su fracaso escolar.

\section{El retorno: Los trámites escolares}

La Sub coordinación regional de la Secretaría de Educación Guerrero, cuenta con un departamento de revalidación que se encarga de hacer las equivalencias a los/ as estudiantes que presenten constancias de estudios. De acuerdo con los lineamientos, los/ as alumnos/as deben traer una boleta binacional o Transfer que tramitan vía consular, con sus constancias de estudio emitidos por la escuela en Estados Unidos y debidamente apostilladas.

En general tanto estudiantes como padres y madres de familia manifiestan que a su regreso a México el ingreso a la escuela es problemático ante la falta de documentación de la escuela de procedencia, del Transfer y del apostille, que dificultan la revalidación de estudios. Por lo general el regreso de familias indocumentadas se da azarosamente como resultado de la deportación y no tienen tiempo de solicitar el historial académico del hijo, ni hacer los trámites en el consulado. Las constancias que traen son solo las papeletas que las escuelas les proporcionaron, su credencial escolar, 0 un documento "informal" elaborado por el profesor o la profesora en Estados Unidos de América. Algunos sólo traen su cartilla de vacunación y su acta de nacimiento de Estados Unidos. Todo esto sin ninguna validez legal para el contexto jurídico mexicano.

Para los trámites de revalidación de estudios en el caso de la secundaria se concentran en la capital del estado, mientras que en el nivel de primaria se hacen localmente mediante una evaluación para asignarles el grado correspondiente en la Sub coordinación Regional.

El conocimiento del PROBEM por parte de las escuelas y las Coordinaciones Regionales es mixto. En regiones con gran demanda de este tipo de estudiantes es relativamente conocido, pero en otras regiones su conocimiento es limitado. Directores y directoras de las escuelas entrevistados/as manifestaron desconocer el surgimiento del Programa, sus objetivos, filosofía y el tiempo que Ileva operando en el estado de Guerrero.

Es importante señalar que en ninguna de los centros educativos visitados en el estado de Guerrero existen publicidad visible del Programa de Educación Binacional México - Estados Unidos (PROBEM), donde se oriente a los padres y madres de familia sobre los requisitos y gestiones que deben cumplir y seguir en la inscripción de sus hijos/as; situación que limita el conocimiento del Programa, su operatividad y bondades del mismo. 


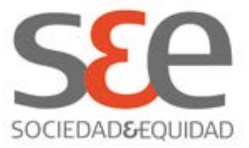

El desconocimiento del programa por los padres y madres de familia, y la no orientación en las escuelas, hace que estudiantes repitan innecesariamente grados ya cursados en Estados Unidos.

El Programa está diseñado para estudiantes en situación migratoria legal y que planeen su regreso a México en condiciones que les permita tramitar su documentación en la escuela y en el Consulado. Esto bajo el supuesto que se conozca la exigencia del Transfer. Sin embargo, la realidad que la investigación encuentra dista mucho del perfil establecido por la norma. El retorno familiar es azaroso y desconocen los requisitos escolares en México. Esto se dificulta más para el estudiante de secundaria que debe tener tiempo y dinero para viajar a la capital, y sobre todo para aquellos estudiantes que quieren ingresar a estudios de preparatoria.

El conocimiento del PROBEM es limitado para la escuela, los profesores y profesoras y desconocido para las familias migrantes en Estados Unidos de América, y para las familias de retorno. Un esfuerzo mayor se impone para los responsables del Programa tanto en su estrategia comunicativa, como en agilizar los trámites de revalidación y adecuar la norma al perfil de estudiantes que retornan sin posibilidades de tramitar la documentación requerida.

\section{El retorno: visión comparativa}

Es evidente que tras una experiencia educativa en Estados Unidos, los/as estudiantes binacionales tengan un acercamiento comparativo de la escuela en México. Su apreciación la hacen a partir de los estándares propios del sistema educativo del país que regresan. Este acercamiento los distingue de sus compañeros/as que al no conocer otra propuesta educativa ven su experiencia natural y única. En su visión diferenciada los/as estudiantes destacan su afronta con los/as docentes, la infraestructura y el equipamiento tecnológico de la escuela.

La educación en Estados Unidos es estatal y no federal como en México. Para la inscripción de sus hijos, los padres deben presentar las vacunas, y probar su lugar de residencia y sus ingresos económicos. El resultado del estudio socioeconómico determinará el costo de la alimentación del estudiante. En el nivel primario, se incluye el transporte escolar.

Los colegios ofrecen programas extracurriculares que van desde clubes deportivos, de matemáticas y ciencias, hasta grupos de pintura, teatro, música y canto. En el último año, se realizan exámenes estatales para determinar el nivel del estudiante.

Sobre la experiencia educativa en Estados Unidos los/ as estudiantes y sus padres y madres la ponderan muy positiva. Manifiestan no haber tenido 


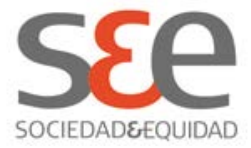

obstáculos para ingresar, recibir buen trato y apoyo de los maestros y compañeros de grupo.

La mayoría de los/ as estudiantes entrevistados tanto de primaria como de secundaria, manifestaron su deseo de seguir estudiando en Estados Unidos, ya que les gustan mucho las escuelas y consideran que se aprende más porque son mejores los/as maestros/as, superior el nivel académico y reciben un trato más respetuoso. Los padres y madres entrevistados coinciden que sus hijos aprenden mejor y están más preparados estudiando en una escuela en Estados Unidos, ponderan la permanencia con alimentación en la escuela, la seguridad y el control sobre los/as estudiantes que les proporciona el tener cámaras de vigilancia y el transporte casa - escuela - casa. Subrayan la actividad extraescolar de los clubes ya que mantienen a sus hijos/as en actividades creativas y los/las protegen de ocios autodestructivos. También afirman desear radicar nuevamente en Estados Unidos para una mejor educación de sus hijos.

La visión comparativa que traen consigo estos/as estudiantes y sus padres y madres también la hacen sobre a infraestructura y el equipamiento tecnológico de la escuela al calificarla de insuficiente y deficiente. En general el profesorado y directivos coinciden con esta observación, pero aún en casos en que la ponderan positivamente, los/as estudiantes, padres y madres de familia difieren. Se hace evidente la distancia entre una visión desde adentro a una comparativa. Los referentes cambian.

La ponderación de la educación norteamericana frente a la mexicana es una constante en los/as estudiantes, padres y madres. La percepción de estos/as estudiantes al considerar que están en un espacio escolar de menor calidad al que asistieron en Estados Unidos, es una fuente más de la frustración y de las dificultades que afrontan.

\section{El retorno: la familia transnacional}

La construcción de una familia transnacional es muy semejante a la historia de Felipe Astudillo quien es un emigrante desde su juventud cuando tuvo que salir de su comunidad a trabajar en otros lados. Primero como jornalero agrícola a la cosecha del jitomate al norte de México en el estado de Sinaloa, después, a finales de los ochenta, hacia los Estados Unidos. Cuando se fue de emigrante internacional y ya estando en aquel país, mandó por su esposa e hijo. Su hijo tenía 7 años, así que lo inscribió para que estudiara en una escuela pública, cursó los seis años de primaria (elementary school) y dos de secundaria (middle school), no teniendo ningún problema para su inscripción al sistema escolar estadounidense. 


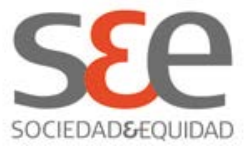

La trayectoria escolar de los/as hijos/as de migrantes es variada. Unos nacen e inician su escolaridad en México; luego migran con sus padres a Estados Unidos en donde se inscriben en la escuela y posteriormente regresan para continuar su escolaridad; esta trayectoria es la más común de todas. Otros nacen en Estados Unidos donde inician su escolaridad y regresan a México a temprana edad para continuarla. Son alumnos/as que pasan de un sistema escolar a otro en más de una ocasión.

En general, tanto estudiantes, maestros/as, directivos y administrativos coinciden en que en la mayoría de los casos el regreso de estos/as alumnos/as es con la mamá, con los hermanos o algún otro familiar. En muy pocos casos de los que se tienen regresa la familia completa, en algunos solo se regresan los hijos con la abuelita o los tíos.

La estancia en los Estados Unidos de los/ as niños/ as y jóvenes migrantes que regresan, oscila entre cuatro y diez años, con una experiencia escolar entre uno y cuatro años. Ellos/ as emigran a la edad de tres a once años, y su retorno va de los diez a dieciséis años. El promedio de cruce en la frontera de estos/ as jóvenes es a lo menos dos veces, y se dirigen hacia diferentes ciudades y condados en los Estados Unidos.

De acuerdo a maestros/as y directivos, el sistema escolar no da cuenta de los alumnos/as que abandonan la escuela para emigrar con sus padres. No existen registros de la ausencia o abandono por este motivo, el cual solo en forma aislada y casual se tiene noticia. El conocimiento de esta situación escolar precaria para el/la estudiante, sólo se observa al regreso y en la solicitud de su reincorporación.

\section{El retorno: la escuela}

La planta docente y directiva de las escuelas de primaria y secundaria adolece de la debida información y preparación de la problemática que los/as alumnos/as binacionales traen consigo. Sin embargo, la experiencia educativa de algunos maestros y maestras han permitido trabajar adecuadamente con estos/ as alumnos.

Maestros/as y directivos entrevistados coinciden en que la planta docente puede estar calificada en tanto se actualice periódicamente y sea completa. Los/as maestros/ as no reciben una preparación especial ni la escuela está adaptada pedagógicamente para este tipo de alumnos/as. Sin embargo existe la disponibilidad de ayudar a los/as alumnos/as binacionales en su adaptación. Sugieren que los/as docentes se capaciten y se especialicen para atender a estos alumnos, y los provean de proyectos, estrategias y materiales didácticos de apoyo con lo cual poder trabajar mejor. 


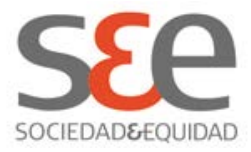

Para subsanar las dificultades que presentan los/as alumnos/as binacionales, algunos directivos de escuelas exigen mayor coordinación con los responsables del PROBEM para su seguimiento escolar. Existe, aunque muy limitado, la Unidad de Servicio de Asistencia Educativa Regular (USAER) que provee de apoyos de psicólogos, terapeutas y trabajadores sociales para tratar problemas de hiperactividad, de aprendizaje y atención de los/ las estudiantes.

En realidad, tanto el estudiantado binacional como los/as docentes implicados/as demandan una asistencia educativa puntual, ya que la investigación identificó casos en el que frente a estudiantes que expresan estados de rebeldía, Ios/as docentes se impacientan y llegan a la agresión física. También se presentaron casos de estudiantes reprobados en la asignatura de inglés por maestros que castigaban la 'insolencia' de ser cuestionados, corregidos y evidenciados en sus limitaciones del conocimiento del idioma. En este contexto escolar, las fortalezas de estos/ as estudiantes se tornan amenazas.

Los problemas propios de los/a niños/as, y jóvenes en situación de estudiantes binacionales y en contextos familiares transnacionales podrían bajar de intensidad si se contara con docentes capacitados y apoyos escolares puntuales que impidan "soluciones" de violencia y mitiguen la segregación y discriminación de la que son objeto por parte de sus compañeros/as.

Es claro que estos/as estudiantes vienen de escuelas con recursos humanos superiores, mejor equipadas tecnológicamente y con experiencias educativas y culturales diferentes. Esto trae consigo identidades cambiantes y visiones comparativas que dificultan su rendimiento escolar y adaptación social.

La no inclusión formal e institucional de la particular problemática de los/as alumnos/as binacionales en la formación escolar normalista de los/as maestros/as, en las prácticas de enseñanza aprendizaje, en las dinámicas y actividades escolares de socialización, permite la invisibilidad del alumno/a binacional que reclama su diversidad y espacio en una escuela no diseñada para contextos plurales sino para homogeneizar culturas y conciencias.

La presencia de estudiantes binacionales en la escuela mexicana exhibe limitaciones y debilidades del sistema educativo y su relato homogéneo que ignora o evade la diversidad cultural que traen consigo el retorno de estos/as alumnos/as. No existe otra opción que adaptarse y producir competencias frente a las nuevas realidades sociales y culturales que la migración y los procesos de integración con Estados Unidos imponen. 


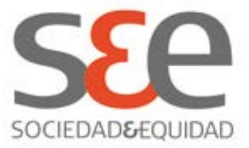

\section{El retorno: la disonancia cognitiva}

Los/ as estudiantes binacionales emprenden nuevos desafíos al inscribirse al sistema educativo mexicano. Ellos/as deben de enfrentar los retos que el ambiente y la escuela les imponen en lo social y lo cultural. Cuatro fueron las principales dificultades detectadas que los/as hacen propensos/as a la deserción: la adaptación o integración social, problemas de aprendizaje, apatía escolar, y la inestabilidad familiar.

Los/ as estudiantes en Estados Unidos no asisten a escuelas bilingües, no les enseñan español, geografía ni historia patria de México. A su regreso esto se califica de preparación académica deficiente y con propensión al fracaso escolar. Sin embargo, docentes y directivos entrevistados subrayan que estos/as estudiantes destacan por sus habilidades en computación e inglés. Pero aún y cuando, ellos/as entienden, hablan, leen, escriben y seguramente piensan en inglés, son obligados a cursar la materia.

En general los/as estudiantes de retorno llegan con un español muy limitado para la lectura y escritura, y en algunos casos para entenderlo y hablarlo. Esto dificulta la asimilación de las lecturas y tareas, afecta su comunicación verbal y la participación en clase, lo que contribuye a su aislamiento y exclusión de los grupos.

La discontinuidad lingüística no solo se da en el espacio escolar sino también en sus casas. Ellos/ as reportan que en su familia hablan ambos idiomas 0 una mezcla de inglés y español; incluso se da el caso que hablan también una lengua indígena. Cabe esperar problemas de aprendizaje, mayor probabilidad de reprobación y apatía escolar, y dificultad por parte de los/ as maestros/as en darse a entender. Algunas madres reportan que sus hijos/ as Ilegan a casa con dudas sobre cuál es la tarea debido a que en las clases no entienden nada.

Al inscribirse en las escuelas, los/ as estudiantes deben auto presentarse en el grupo mencionando la escuela de procedencia. Este es el primer filtro en la que ellos/as se sienten discriminados/as por la risa de los/as compañeros/ as ante sus dificultades de expresión oral. Esto hace una diferencia en el grupo, crea problemas de comunicación y es fuente posible de discriminación y desadaptación.

La escuela no está preparada para reconocer y proponer las acciones pedagógicas necesarias para la discontinuidad lingüística que viven los/as alumnos/as binacionales a su regreso escolar en México. De acuerdo a directivos y docentes, la escuela no contempla dinámicas de inclusión escolar y social de los/as jóvenes estudiantes migrantes, no existe ninguna actividad relacionada con la bienvenida, el rechazo o la discriminación social al que 


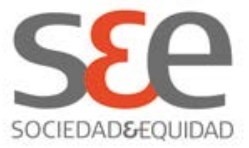

pueden estar expuestos/ as en la escuela. Estas actividades dependen de cada profesor/a.

En realidad, la ausencia de actividades de integración, se dan por el hecho de que el sistema escolar y la institución ignoran la existencia de estos/as estudiantes. La mayoría de los/as docentes entrevistados/as desconocían la presencia en sus cursos de alumnos/as binacionales, igual con los directivos de las escuelas. Su invisibilidad la expresa muy bien un profesor de matemáticas: "cuando recibimos estos alumnos muchas veces llegan desorientados, hablando muy poco español y con miedo y se toma como algo normal como se fueran de aquí todos".

La dificultad de integración social se expresa también en las actividades de ocio que no comparten. Algunos/as estudiantes extrañan el beisbol como deporte en un espacio donde es el futbol lo que se juega, otros/as expresan su gusto por el rock en especial con el Hip hop que no lo disfrutan con sus compañeros/ as de escuela que les gustan más "Ios corridos" y "Ia bachata"

La Directora de una escuela, enfatiza la situación familiar dislocada en la que viven estos estudiantes para explicar las dificultades de adaptación social, los problemas de aprendizaje y la propensión al abandono de sus estudios.

Estas dificultades comunes a los/ as estudiantes de retorno, se complican con estados de rebeldía cuando se trata de adolescentes en secundaria. Al respecto una maestra señaló que los/as estudiantes emigrantes son muy rebeldes, no le hacen caso al maestro. Se le pregunta por las razones de esta rebeldía y la educadora respondió:

No sé si porque no entienden el español y tenemos dificultad en hacernos entender o porque son educados con otras costumbres allá en Estados Unidos. Aquí se les inculcan los valores de la familia, donde el papá y la mamá son pilares del hogar, de la familia ampliada y el respeto hacia sus miembros. Todo esto, tal parece no lo toman mucho en cuenta en el país vecino, lo que implica un cambio en los valores del estudiante binacional. Lo cierto es que por su situación familiar de migrar, no aprenden ni aquí ni allá, porque están por un año aquí y luego se los vuelven a llevar, son ellos los que sufren estos cambios.

Es obvio que el relato sobre la "familia normal, convencional" que la escuela reproduce crea una tensión con la experiencia íntima familiar del y la estudiante binacional que lo/a sumerge en un estado de incompatibilidad cognitiva que impacta sus creencias y actitudes. El manejo o negociación de esta incongruencia o disonancia cognitiva (Festinger, 1957), demanda 


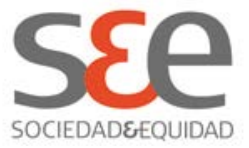

competencias y motivaciones que el/la estudiante carece y la escuela no provee.

La disonancia cognitiva en estos estudiantes se refuerza en la consideración de sí mismos/ as como México - americanos/ as.

La situación de estos/as estudiantes binacionales hace que sus identidades sean cambiantes y frágiles, con problemas de adaptación con su entorno en una edad en la que están construyendo una identidad que les permita su inserción social.

El aprendizaje del idioma, la integración y rendimiento escolar para aquellos alumnos/as que retornan junto con sus padres y/o madres, y que no reinciden en movimientos migratorios, es por lo general, cuestión de tiempo. Ellos/as se integran fácilmente con los deportes o incluso a través de la religión.

\section{Conclusiones}

La investigación sobre los/as alumnos/as binacionales de retorno en situación familiar transnacional, permite visualizar en México nuevos/as actores/as sociales que emergen al seno del mayor, más amplio y antiguo conflicto social: la pobreza. La familia transnacional y los/as estudiantes binacionales son resultado de la particular migración y relación laboral con un país limítrofe que ofrece oportunidades de estudio y una vida digna que no encuentran en su terruño.

La experiencia educativa y cultural de estos/ as estudiantes, su geografía emocional, su paisaje dislocado familiar, su discontinuidad lingüística, la consideración de sí mismos como mexicano/a - americano/a, y su enfoque interpretativo, surgen con fuerza diferenciada a su retorno obligado, sumergiéndolos en estados de disonancia cognitiva frente a una escuela ajena a la diversidad y reproductora de identidad nacional.

Los/as alumnos/as binacionales poseen fortalezas que el sistema educativo y la formación docente ignoran, o los perciben como amenaza. Ellos/ as son la primera generación de la migración de retorno en el seno de un sistema escolar que los/as invisibiliza y obliga a integrarse a cambio de autonegación, riesgo de exclusión y fracaso escolar.

Este estudio evidencia una realidad que no se puede soslayar. El hecho migratorio adquiere cada vez mayor presencia, amplía y profundiza la relación binacional México - Estados Unidos, y es promotor de cambios. Las instituciones y en especial las educativas en México, diseñan políticas y reproducen relatos para un universo familiar y nacional que ya no existen. Ignoran y tienen 


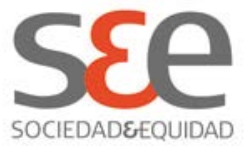

dificultad para pensar que lo que sucede en la frontera modifica todos los procesos sociales y nos inserta a todos en el transnacionalismo.

\section{Referencias Bibliográficas}

Basch, I. Glick Schiller, N. y B. Szanton (1994). Nations Unbound: Transnational Projects, Postcolonial Predicaments and Desterritorilized. New York: Gordon and Breach Publishers.

Bryceson, D. \& Vuorela, U. (2002). The transnational family new European frontiers and global networks. Oxford, 0: University Press.

Di Leonardo, M. (1992). The female world of cards and holidays: Women, families, and the work of kinship. In: T. Barrie \& M. Yalom (Ed.), Rethinking thefamily: Somefeminist questions (pp. 246-261). Boston, MA: Northeastern University Press.

Durand, Jorge y Douglas S. Massey (2003). Clandestinos, migración MéxicoEstados Unidos en los albores del siglo XXI. Miguel Ángel Porrúa, México.

Giménez, G. (2010). Materiales para una teoría de identidades sociales. Instituto de investigaciones sociales de la Universidad Autónoma de México, México. [Documento en línea], disponible desde internet en: $<w w w$. mexicanosdisenando. org. $\mathrm{mx} /$ articulos. php?artipo $=2-31 \mathrm{k}>$

Faist, T. (2000). The Volume and Dynamics of International Migration and Transnational Social Spaces. Oxford, O: Oxford University Press.

Festinger, L. (1957). A theory of cognitive dissonance. Stanford University Press, Stanford, California.

Fundación BBVA Bancomer y el Servicio de Estudios Económicos, México de BBVA Research (2012). Situación Migración Mexicana. BBVA, Julio 2012, Mexico. Disponible desde internet en: $\varangle$ www. bbvaresearch. com>.

Guarnizo, E. (2006). Migración, globalización y sociedad: teorías y tendencias en el siglo XX. En G. Ardila Colombia: Migraciones, transnacionalismo y desplazamiento. Bogotá: Colección CES.

Hondagneu-Sotelo, P. \& Ávila, E. (1997). “I'm Here, but I'm There: The Meanings of Latina Transnational Motherhood". Gender and Society (Kansas, USA), 11 (5). 


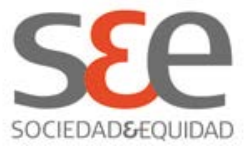

Levitt, P. (1998). Social Remittances: “Migration Driven Local-Level Forms of Cultural Diffusion". International Migration Review (New York, USA), 32 (4), pp. 926-948.

Levitt, P. (2001). The transnational Villagers. Berkeley y Los Ángeles: University of California Press.

Parella S. \& Cavalcanti. L. (2006, noviembre). “Una aproximación cualitativa a las remesas de los inmigrantes peruanos y ecuatorianos en España y su impacto en los hogares transnacionales". Revista Española de Investigaciones Sociológicas, (116), pp. 241-257.

Pedone, C. (2007, marzo). Los/ as hijos/ as de la migración ecuatoriana: lecturas transnacionales de los cambios familiares. Comunicación presentada en el $\mathrm{V}$ Congreso sobre la Inmigración en España. Migraciones y Desarrollo Humano, Valencia, España. Universitat De València, Ceim - Centro de Estudio para la Integración Social y Formación de Inmigrantes-. [Documento en línea], disponible desde internet en: \ttp:// www. migrastudium. org/ doc/ LRecolons\%20Transnacionalismo\%20y\%20re des\%20migratorias\%20VCongreso2007.doc>

Pribilsky, J. (2004). "Aprendemos un convivir: las relaciones conyugales, la copaternidad, y la vida familiar entre los migrantes transnacionales en la ciudad de Nueva York y los Andes ecuatorianos". Global Neworks (Oxford, Reino Unido), 4 (3).

Secretaría de Educación del estado de Guerrero SEG, Subsecretaría de Planeación Educativa (2010). Estadísticas de estudiantes adscritos al Programa Binacional de Educación Migrante México-EUA (PROBEM), Guerrero, México.

Schiller, N. Bash, L. \& Szaton, C. (1992). "Towards a Transnational Perspective in Migration: race class ethnicity and nationalism reconsidered". Annals of the New York Academy of Sciences 645, New York, USA.

Wimmer, A y N. Glick Schiller (2003). "Methodological Nationalism, the Social Sciences, and the Study of Migration: an Essay in Historical Epistemology". International Migration Review. 37(3):576-610.

Woo Morales, O. (2001). Las mujeres también nos vamos al Norte. Universidad de Guadalajara, México. 


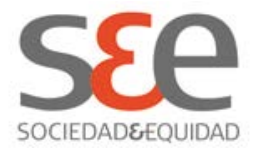

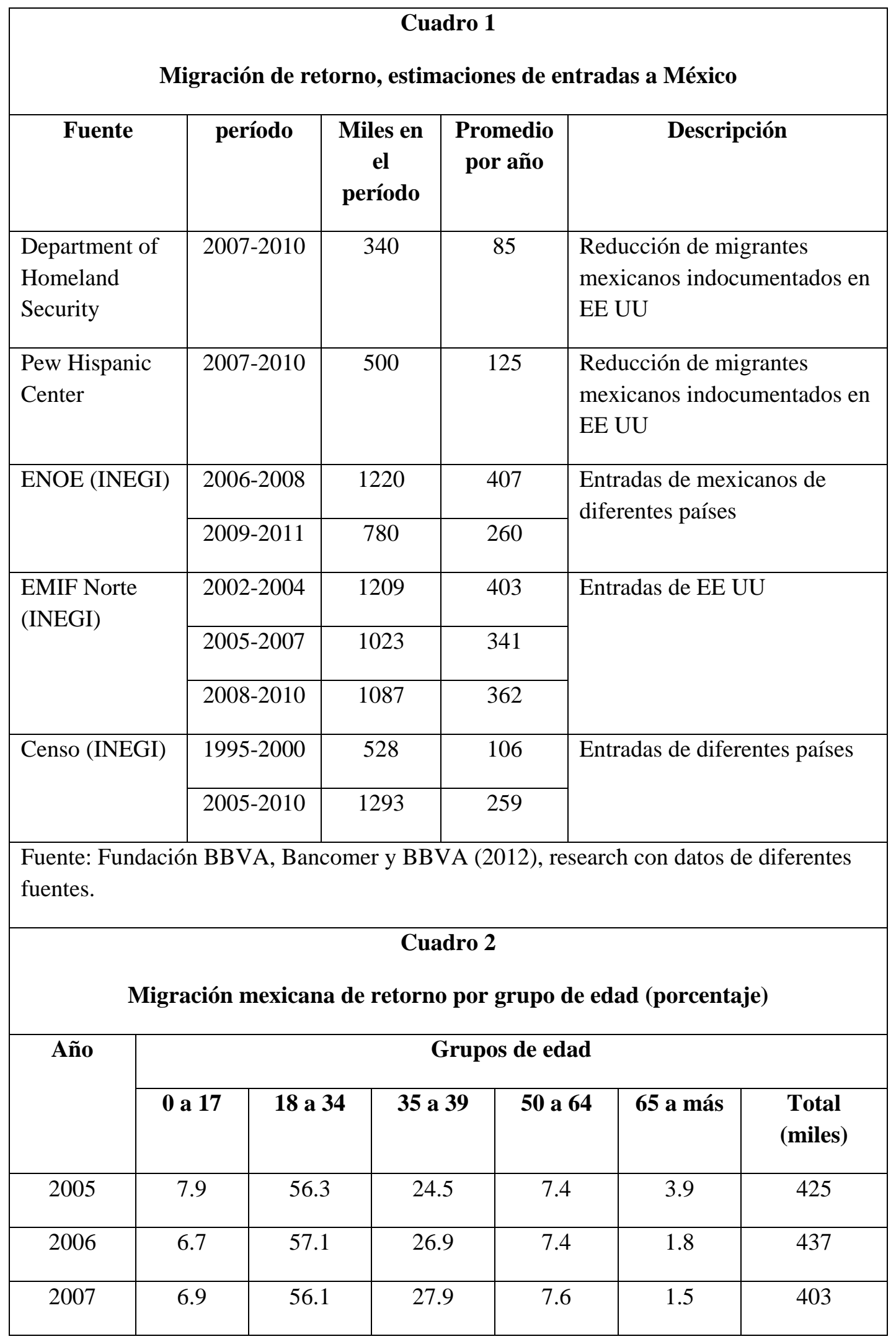




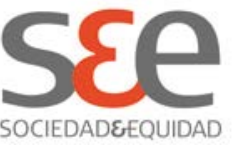

\begin{tabular}{|l|c|c|c|c|c|c|}
\hline 2008 & 6.9 & 57.7 & 25.8 & 7.7 & 2.0 & 379 \\
\hline 2009 & 8.8 & 52.6 & 28.7 & 7.0 & 2.9 & 329 \\
\hline 2010 & 8.2 & 54.6 & 26.7 & 8.5 & 2.0 & 264 \\
\hline 2011 & 6.2 & 56.5 & 22.3 & 11.3 & 3.7 & 202 \\
\hline
\end{tabular}

Fuente: Fundación BBVA, Bancomer y BBVA (2012), research, estimaciones a partir de la construcción de paneles de la ENOE, 2005-2011, con la metodología de migrantes internacionales del INEGI.

\section{Cuadro 3}

Estudiantes de primaria y secundaria atendidos en el estado de Guerrero, México por el PROBEM. Ciclo escolar 2005 - 2006 y 2008 - 2009

\begin{tabular}{|c|c|c|c|c|c|c|c|}
\hline \multicolumn{4}{|c|}{ Estudiantes atendidos } & \multicolumn{4}{c|}{ Porcentaje: variación } \\
\hline \multicolumn{2}{|c|}{ Primaria } & \multicolumn{2}{|c|}{ Secundaria } & \multicolumn{2}{c|}{ Primaria } & \multicolumn{2}{c|}{ secundaria } \\
\hline $2005-$ & $2008-$ & $2005-$ & $2008-$ & $2005-$ & $2008-$ & $2005-$ & $2008-$ \\
2006 & 2009 & 2006 & 2009 & 2006 & 2009 & 2006 & 2009 \\
\hline 427 & 471 & 83 & 180 & 100 & $10.3 \%$ & 100 & $116.9 \%$ \\
$(83.7 \%)$ & $(72.4 \%)$ & $(16.3 \%)$ & $(27.6 \%)$ & & & & \\
\end{tabular}

